\title{
Vacancy-Hydrogen Complexes in Germanium
}

\author{
B. J. Coomer ${ }^{\mathrm{a}, 1}$, P. Leary ${ }^{\mathrm{a}}$, M. Budde ${ }^{\mathrm{b}}$, B. Bech Nielsen ${ }^{\mathrm{b}}$, \\ R. Jones ${ }^{\mathrm{a}, 1}, \mathrm{~S}$. Öberg ${ }^{\mathrm{c}, 2}$ and P. R. Briddon ${ }^{\mathrm{d}}$ \\ ${ }^{a}$ Department of Physics, The University of Exeter, Exeter, EX4 4QL, \\ United Kingdom \\ bInstitute of Physics and Astronomy, Aarhus University, DK-80000, Aarhus, Denmark \\ c Department of Mathematics, University of Luleå, Luleå, S95 187, Sweden \\ ${ }^{\mathrm{d}}$ Department of Physics, The University of Newcastle upon Tyne, \\ Newcastle upon Tyne, NE1 7RU, United Kingdom
}

\begin{abstract}
Local-density-functional pseudopotential theory is used to investigate the structural, electronic and vibrational properties of vacancy-hydrogen complexes in germanium. The results are compared with recent infrared absorption data from proton and deuteron implanted Ge. The acceptor and donor levels of the $\mathrm{VH}_{n}$ defects are derived semi-empirically from the relaxed structures.
\end{abstract}

Key words: $\mathrm{Ge}, \mathrm{H}$, vacancy, complexes, Ab Initio theory

\section{Introduction}

The tendency for the vacancy to act as a trap for hydrogen has been well studied in many semiconductors over recent years. The structure and vibrational properties of vacancy-hydrogen complexes in silicon have been systematically investigated both theoretically[1-3] and experimentally[1,5,4] resulting in reliable assignments of many of the local vibrational modes. We discuss the results of infrared experiments on ion-implanted $\mathrm{Ge}$ and ab initio modelling. A full report on techniques used will be published at a later date.

1 EPSRC and the ENDEASD network are thanked for computer resources and financial support respectively.

2 Financial support by NFR and TFR, and computer resources by PDC at KTH in Sweden are gratefully acknowledged. 


\section{Results}

The relative frequencies of the vibrational stretch modes of the $\mathrm{VH}_{n}, \mathrm{n}=2,3,4$ defects in germanium were observed to be very similar to those identified in silicon with a constant factor mapping Si stretch frequencies onto their counterparts in Ge. The vibrational modes for $\mathrm{VH}$ in Ge have not yet been observed. There also remains some doubt as to the assignment of the stretch modes to $\mathrm{VH}_{3}$. It is uncertain whether these modes arise from $\mathrm{VH}_{3}$ or $\mathrm{V}_{2} \mathrm{H}_{6}$. The annealing data shows these modes to arise from a defect which is more stable than $\mathrm{VH}_{4}$ which may suggest that they originate from $\mathrm{V}_{2} \mathrm{H}_{6}$ rather than $\mathrm{VH}_{3}$.

The calculated local vibrational modes (LVM's) for the neutral charge states of the $\mathrm{VH}_{n}$ complexes and the effects of deuteration are compared with experiment in Table 1. The assignments of those vibrational modes arising from mixed isotopic defects are tentative.

Neutral VH gives rise to a partially occupied electronic doublet in the forbidden gap. The resulting Jahn-Teller distortion to $\mathrm{C}_{1 h}$ symmetry is effected by a rebonding by pairs of the Ge dangling bonds across the gap. The reconstructed bond length in the relaxed defect was calculated to be $2.78 \AA$ in the relaxed structure (cf. $2.4 \AA$ for bulk Ge). The high electron density in the region between the two paired atoms repels the $\mathrm{H}$ atom so that the $\mathrm{Ge}-\mathrm{H}$ bond moves $4^{\circ}$ out of the [111] axis. The $\mathrm{Ge}-\mathrm{H}$ bond was found to be $1.541 \AA$ as compared to $1.52 \AA$ in molecular $\mathrm{GeH}_{4}$. The Ge atom bonded to the hydrogen moves outwards from the vacancy by $0.32 \AA$ along [111].

The relaxed dihydrogen-vacancy defect was found to have $\mathrm{C}_{2 v}$ symmetry. The reconstructed Ge-Ge bond length was found to be $2.79 \AA$. The effect of the additional $\mathrm{H}$ atom in the defect results in a shortening of the Ge-H bond length to $1.531 \AA$. The symmetry of the $\mathrm{VH}_{3}$ defect was assumed to be $\mathrm{C}_{3 v}$. The relaxed defect then has three equivalent $\mathrm{H}$ atoms each with a Ge-H bond length of $1.536 \AA$. The angle made between the Ge-H bonds and the $<111>$ directions was found to be $6.2^{\circ}$. An orbital-singlet level was found deep in the gap occupied by a single electron.

$\mathrm{VH}_{4}$ has four equivalent $\mathrm{H}$ atoms ( $\mathrm{T}_{d}$ symmetry) each bonded to a Ge atom with a bond length of $1.526 \AA$ in $\mathrm{VH}_{4}$. The Ge atoms move outwards from the vacancy by $0.33 \AA$ along [111] from their ideal sites. $\mathrm{VH}_{4}$ was found to possess an energy gap free from defect levels.

Also calculated were the vibrational modes for both $\mathrm{VH}_{3}^{-}$and neutral $\mathrm{V}_{2} \mathrm{H}_{6}$. The effect of the addition of an electron to $\mathrm{VH}_{3}$ was to shorten all $\mathrm{Ge}-\mathrm{H}$ bond lengths by $0.7 \%$ resulting in modest increases in the frequencies of the $\mathrm{E}$ and $\mathrm{A}$ symmetry vibrational modes by 6 and $25 \mathrm{~cm}^{-1}$ respectively. $\mathrm{V}_{2} \mathrm{H}_{6}$ was found to have the shortest Ge-H bond length of $1.52 \AA$. Two stretch modes of $\mathrm{A}_{2 u}$ and $\mathrm{A}_{1 g}$ symmetry were found at 2131 and $2129 \mathrm{~cm}^{-1}$ respectively. Two doublet $\mathrm{E}_{u}$ and $\mathrm{E}_{g}$ modes 
were found at 2128 and $2120 \mathrm{~cm}^{-1}$ respectively. The $\mathrm{A}_{1 g}$ and $\mathrm{E}_{g}$ modes are infrared inactive. The splitting between the I-R active $\mathrm{A}_{2 u}$ and $\mathrm{E}_{u}$ modes was found to be $3 \mathrm{~cm}^{-1}$, substantially smaller than that calculated difference between the $\mathrm{A}$ and $\mathrm{E}$ modes in $\mathrm{VH}_{3}$.

Preliminary calculations using a method described elsewhere[7] were used to find the acceptor and donor levels. Acceptor levels were calculated for $\mathrm{VH}, \mathrm{VH}_{2}, \mathrm{VH}_{3}$ and were all found to lie within $0.35 \mathrm{eV}$ of the valence band edge. The donor levels were calculated for $\mathrm{VH}$ and $\mathrm{VH}_{3}$ and lie close to the valence band .

The reorientation barrier was calculated by optimising the energy of a $\mathrm{VH}_{3}$ defect with one of the $\mathrm{H}$ atoms constrained to be equidistant from two unhydrogenated $\mathrm{Ge}$ atoms. The difference in energy between this configuration and the unconstrained $\mathrm{VH}_{3}$ was calculated to be $0.30 \mathrm{eV}$.

\begin{tabular}{|c|c|c|c|c|c|}
\hline & VH & VD & & & \\
\hline & $2017(?)$ & $1435(?)$ & & & \\
\hline & $\mathrm{VH}_{2}$ & VHD & $\mathrm{VD}_{2}$ & & \\
\hline $\mathrm{s}$ & 2102(1993) & 2090(?), 1486(1437) & $1495(1444)$ & & \\
\hline $\mathrm{a}$ & 2078(1980) & & $1478(1433)$ & & \\
\hline & $\mathrm{VH}_{3}$ & $\mathrm{VH}_{2} \mathrm{D}$ & $\mathrm{VHD}_{2}$ & $\mathrm{VD}_{3}$ & \\
\hline $\mathrm{s}$ & $2098(2025)$ & $2088(2023), 1477(1456)$ & 2077(2018), 1484(1461) & $1491(1465)$ & \\
\hline $\mathrm{a}$ & $2067(2015)$ & 2067(2013) & $1471(1453)$ & $1471(1454)$ & \\
\hline & $\mathrm{VH}_{4}$ & $\mathrm{VH}_{3} \mathrm{D}$ & $\mathrm{VH}_{2} \mathrm{D}_{2}$ & $\mathrm{VHD}_{3}$ & $\mathrm{VD}_{4}$ \\
\hline $\mathrm{s}$ & 2187(-IR) & 2168(2084) & $2149(2080)$ & $2128(2075)$ & \\
\hline $\mathrm{a}$ & $2107(2062)$ & $2107(2062$ or 2066$)$ & 2107(2066),1499(1486) & $1499(1487)$ & 1499(1489) \\
\hline $\mathrm{s}$ & & 1511(1491) & $1524(1499)$ & 1539 & 1554(-IR) \\
\hline
\end{tabular}

Table 1

Calculated and observed stretch frequencies of $\mathbf{V H}_{n} \mathbf{D}_{m}$. Experimental frequencies are given in parentheses. Assignments of experimental absorption lines to $\mathrm{VH}_{2} \mathrm{D}, \mathrm{VHD}_{2}$, $\mathrm{VH}_{3} \mathrm{D}, \mathrm{VH}_{2} \mathrm{D}_{2}, \mathrm{VHD}_{3}$ are tentative. s=total symmetric, $\mathrm{a}=$ non-total symmetric, $\mathrm{IR}=$ infrared inactive.

\section{Concluding remarks}

In summary, the vibrational modes were found to be between 99 and $95 \%$ of the observed data. The errors were found to increase for the defects with fewer hydro- 
gen atoms. Using this trend we predict that the $\mathrm{VH}$ defect will possess an $\mathrm{A}_{1}$ mode at around $1885 \mathrm{~cm}^{-1}$.

The infrared active stretch modes for $\mathrm{V}_{2} \mathrm{H}_{6}$ are found at higher frequency than those in $\mathrm{VH}_{3}$ by a modest amount $(<2 \%)$. We expect the stretch modes $\mathrm{VH}_{3}^{-}$to be shifted upwards in frequency with respect to neutral $\mathrm{VH}_{3}$.

The Kohn-Sham levels show a similar pattern to that found in silicon : the addition of hydrogen atoms acts to move the defect levels from the gap. All the calculated acceptor and donor levels are predicted to lie in the lower half of the bandgap.

The calculated adiabatic barrier for the reorientation of $\mathrm{VH}_{3}$ is somewhat larger than the observed barrier in silicon $(87 \mathrm{meV})$. We therefore propose that the movement of the hydrogen atom across the $\mathrm{VH}_{3}$ defect is effected by tunneling from an excited state similar that described for the reorientation of $\mathrm{O}_{2}^{-}$in $\mathrm{KI}[6]$.

\section{References}

[1] B.Bech Nielsen, L. Hoffmann, M. Budde, R. Jones, J. Goss, S. Oberg, Mat. Sci. For. 196, 933 (1995).

[2] S. K. Estreicher, Mater. Sci. Engr. Reports 14, 317 (1995).

[3] H. Xu, Phys. Rev. B 46, 1403 (1992).

[4] P. Stallinga, P. Johannesen, S. Herström, K. Bonde Nielsen and B. Bech Nielsen, Phys. Rev. B (1998), in press.

[5] L. M. Xie ,M. W. Qi and J. M. Chen, J. Phys. Cond. Mat. 3, 8519 (1991).

[6] R. H. Silsbee, J.Phys. Chem. Solids. 28 , 2525 (1967).

[7] R. Jones, A. Resende, S. Öberg and P. R. Briddon, Proceedings of the E-MRS Spring Meeting, 16-19 June 1998 Strasbourg, France (Submitted). 